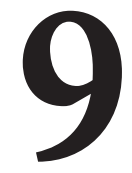

\title{
LAS CARAS DEL PLAGIO INCONSCIENTE EN LA ESCRITURA ACADÉMICA
}

(THE FACES OF UNCONSCIOUS PLAGIARISM IN ACADEMIC WRITING)

Mari Mar Boillos Pereira

Universidad del País Vasco / Euskal Herriko Unibertsitatea

DOI: 10.5944/educXX1.25658

\section{Cómo referenciar este artículo/How to reference this article:}

Boillos Pereira, M.M. (2020). Las caras del plagio inconsciente en la escritura académica. Educación XX1, 23(2), 211-229, doi: 10.5944/educXX1.25658

Boillos Pereira, M.M. (2020). The faces of unconscious plagiarism in academic writing. Educación XX1, 23(2), 211-229, doi: 10.5944/educXX1.25658

\section{RESUMEN}

El plagio es un fenómeno de naturaleza compleja con cada vez más presencia en el mundo universitario. Escribir un texto académico exige el uso de ideas, datos, etc. ajenos y el riesgo de que esas fuentes no estén correctamente referenciadas y de que las ideas se plasmen sin el debido reconocimiento a su autor es elevado. Sin embargo, es necesario distinguir entre el plagio deliberado, el realizado con pleno conocimiento de su gravedad, y el plagio inconsciente, aquel que se produce por falta de conocimiento de uso de las fuentes. En este estudio se analizan las producciones escritas de 100 estudiantes de primer curso universitario del grado en Educación Primaria. Se trata de unas actas de congreso fruto de un curso en el que se ha seguido la metodología basada en proyectos y cuya finalidad es el desarrollo de la competencia comunicativa académica de los estudiantes. Estos han sido instruidos en el sistema de citación APA. El análisis se ha 
realizado a partir del estudio cualitativo de los informes de un software de detección de plagio. El fin no es otro que observar los usos de la información que podrían ser considerados plagio y que se deben, en realidad, a una falta de conocimiento de estrategias de uso de la información. Este análisis da lugar a la creación de una taxonomía de casos de plagio inconsciente. Los resultados obtenidos serán clave a la hora de diseñar propuestas para la formación del alumnado en escritura académica.

\section{PALABRAS CLAVE}

Escritura académica; Educación superior; alfabetización académica; plagio.

\section{ABSTRACT}

Plagiarism is a complex phenomenon with a growing presence in academia. Writing an academic text requires the use of other people's ideas, data, etc. and the risk that these sources are not correctly referenced or that the ideas are reflected without due recognition to its author is high. However, it is necessary to distinguish between deliberate plagiarism, carried out with full knowledge of its severity, and unconscious plagiarism, which occurs due to lack of knowledge of the use of sources. This study analyzes the written productions of 100 freshmen in the Degree of Primary Education. These are conference proceedings resulting from a project-based methodology course that aimed to develop students' academic communicative competence. They received instruction on the APA citation system. The analysis is based on the qualitative study of the reports by plagiarism detection software. The purpose is to observe the use of information that could be considered plagiarism but are due to a lack of strategies for the use of information. This analysis leads to the creation of a taxonomy of cases of unconscious plagiarism. The results obtained will be key when designing proposals for the training of students in academic writing.

\section{KEYWORDS}

Academic writing; Higher Education; academic literacy; plagiarism. 


\section{INTRODUCCIÓN}

La escritura de textos en el ámbito académico exige el uso de ideas, datos, etc. de otros autores. En todos los casos, estas ideas deben estar debidamente referenciadas y su autoría debe ser explicitada si no se quiere incurrir en la práctica fraudulenta conocida como plagio. Hoy en día, el acceso casi ilimitado a los textos científicos a través de la red y el nacimiento de herramientas para la detección de los casos de plagio ha puesto sobre la mesa el debate acerca de qué medidas adoptar para que esta práctica no se convierta en un modo de actuar generalizado en el ámbito académico.

En este contexto, son numerosos los estudios que se han centrado en el estudio de dicho fenómeno desde diversos prismas. Existen, por ejemplo, análisis del porcentaje de plagio que hay en los textos escritos por estudiantes universitarios (Comas, Sureda, y Oliver, 2011). También, se ha mostrado un interés por conocer la percepción que los propios estudiantes manifiestan acerca de esta práctica (Adam, Anderson, \& Spronken-Smith, 2017; Childers $\&$ Bruton, 2016). Incluso se ha puesto el foco en el impacto que Internet ha tenido en el incremento de este ejercicio (Domínguez, Pérez y Quiroz, 2016 e Ison, 2015). Sin embargo, existe el reto pendiente de conocer mejor cuáles son los motivos que traen consigo la existencia de estos casos, así como el reto de distinguir entre aquellos que se producen de manera optativa y los que entrañan una casuística diferente y son de tipo involuntario.

El interés por conocer más acerca de la naturaleza de esta problemática es el embrión del objetivo principal de este estudio. El propósito es analizar las producciones de los estudiantes universitarios y discriminar los casos de plagio inconsciente de los casos de plagio deliberado con la finalidad de crear una taxonomía de usos de la información que dan lugar a lo que se considerará plagio inconsciente. Esta taxonomía permitirá reflexionar acerca de qué medidas se pueden adoptar a nivel educativo para evitar estas circunstancias.

De ahí que, en primer lugar, se proponga una definición de qué se entiende por plagio en este trabajo y se profundice en la distinción entre plagio deliberado y plagio inconsciente, así como en la razón de su existencia. Posteriormente, se expondrá la metodología empleada en este estudio, las preguntas de investigación que lo han guiado y una descripción del procedimiento de análisis de los datos. Finalmente, se presentarán los resultados obtenidos y se reflexionará acerca de su validez y utilidad para la formación en escritura académica del alumnado que se inicia en el ámbito universitario. 


\section{EL PLAGiO}

Copia, robo, usurpación de la propiedad intelectual...son algunos de los vocablos que se han asociado tradicionalmente al concepto de plagio. En términos generales, el plagio se entiende como el uso de la información de otros textos sin que estos hayan sido debidamente referenciados de manera explícita. El Diccionario de la Real Academia Española (2019) habla de plagio como el acto de "copiar en lo sustancial obras ajenas, dándolas como propias", pero los estudios actuales muestran que para poder hablar de esta realidad, es importante entender en primer lugar el concepto en toda su complejidad.

Pecorari (2008) afirma que para que haya plagio puede haberse dado cualquiera de estas circunstancias: que se reproduzcan ideas o palabras presentes en la fuente, que se repitan frases de la fuente, que no se referencie el texto correctamente o que se atribuya de manera errónea una autoría. Walker (2010), en esta misma línea, distingue tres tipos de plagio. El primero, el verbatim, hace referencia a una reproducción palabra por palabra sin que se indique la autoría del documento. El segundo, el purloining es la copia indiscriminada de otro trabajo; es decir, la reproducción de parte o de la totalidad de un trabajo ajeno como propio. Por último, con sham paraphrasing se alude a los casos en los que se utiliza la misma información que en la fuente original con ligeras modificaciones, pero sin que se esta se cite debidamente.

Otros autores como McBride (2012) han centrado su atención en el rol que cumple el parafraseo en los casos de plagio. El denominado como patchwriting supone la modificación de ciertos elementos del texto original: cambio de verbos, uso de sinónimos, alteración en el orden de la oración... que, por resultar insuficiente, pueden ser identificados como casos de plagio. Sin embargo, el patchwriting podría considerarse también una estrategia de escritura, siempre y cuando la modificación del texto original sea suficiente y se produzca un reconocimiento de las fuentes (Vargas-Franco, 2019).

Además de los diferentes tipos de plagio que existen, es importante destacar que el plagio es un fenómeno multicausal que tiene su origen en dificultades de distinta naturaleza. Entre ellos, se encuentran factores de tipo externo y de tipo interno.

En cuanto a los factores de tipo externo, la universidad ocupa un lugar destacado. La sobrecarga académica, la ausencia de suficientes orientaciones docentes o la naturaleza de las tareas que se demandan podría decirse que propician la aparición de casos de plagio (Hunt, 2003; Sureda, Comas, y Urbina, 2005). La velocidad a la que se pide que el alumnado produzca 
los trabajos y el hecho de demandar año tras año los mismos quehaceres podría haber dado lugar al denominado fast food académico en el que el plagio es un integrante más (Linne, 2014). Aquí entran en juego, también, las metodologías de enseñanza-aprendizaje empleadas por los docentes. Estas se caracterizan por no ofrecer acompañamiento durante el proceso de génesis del texto y por no promover la conciencia de la importancia del uso de la información desde las diferentes asignaturas (Vargas-Franco, 2019). De la misma manera, autores como Bloch (2012) hablan de la ausencia de prácticas en las que los estudiantes tienen la oportunidad de desarrollar una voz propia en el discurso.

Entre los factores externos y, concretamente, los que tienen una dimensión social, parece reseñable la masiva distribución de información que tiene lugar en la red y el acceso a fuentes cada vez más ilimitadas que podrían favorecer la reproducción indiscriminada de ideas (Jaramillo y Rincón, 2014). En la actualidad, existen, incluso, páginas en las que se cuelgan trabajos universitarios completos con libre acceso que facilitan más, si cabe, estas prácticas. Otra pieza del puzle la ocupan las familias: la presión que ejercen ante el estudiante, las expectativas que generan y el miedo al fracaso de sus allegados pueden derivar, asimismo, en estas prácticas (Amiri \& Razmjoo, 2016). Por último, cabe reseñar la importancia de la cultura y es que no todas entienden la reproducción literal de ideas de la misma manera. De hecho, en oriente la copia textual es considerada como algo positivo ya que se entiende que el aprendizaje se produce a través de un proceso de memorización y posterior reproducción (Adam, Anderson, \& Spronken-Smith, 2017).

Entre los factores de tipo interno, tienen relevancia las características de tipo personal como son el deseo de buenas notas, la vaguedad o el miedo a pedir ayuda (Amiri \& Razmjoo, 2016). De igual modo, podrían incluirse en este grupo la falta de motivación o la falta de autoestima que puede llevar al estudiante a pensar que las ideas propias no merecen tener su espacio en el ámbito académico (Ochoa y Cueva, 2014). En este sentido, ocupa un lugar destacado la percepción que los propios autores tienen acerca de lo que consideran que es y no es plagio (Childers y Bruton, 2016) y otras cuestiones asociadas a la ética individual.

Serían factores de tipo interno, también, las prácticas llevadas a cabo por el escritor/estudiante. Entre ellas estarían las lecturas superficiales y poco críticas o, incluso, la idea de que el docente no se leerá la totalidad de los trabajos (Ochoa y Cueva, 2014). Comas, Sureda y Oliver (2011) consideraban, además, que existen serios problemas de integridad académica. En su investigación demostraron que un 70\% de los estudiantes universitarios, en algunos casos, incluso, porcentajes superiores, afirmaba 
haber realizado prácticas fraudulentas de diferente naturaleza. Estos porcentajes se atestiguan, principalmente, en los dos primeros años de la formación académica (Comas, Sureda, y Oliver, 2011). No hay que olvidar tampoco los modos más frecuentes de búsqueda de la información en Internet: búsquedas solo en buscadores generales como Google, solo en la lengua materna del estudiante y búsquedas en las que no existe planificación previa (Egaña, 2012). En algunos casos, estas pueden ser actitudes heredadas de la etapa educativa previa y consecuencia de los modelos educativos a los que han sido expuestos.

No obstante, entre las causas que dan lugar a plagio, es necesario valorar que, en ocasiones, estas se producen o, al menos, así las identifican los software para el reconocimiento del plagio, sin que el estudiante sea capaz de identificar si lo que está haciendo es o no plagio. En estos casos, el plagio no es una cuestión de mala fe o de falta de integridad académica, sino que tiene lugar por una falta de conocimiento de los recursos que están a su disposición para no llevar a cabo esta práctica.

Como consecuencia, podrían identificarse dos tipos de plagio: el plagio deliberado y el plagio inconsciente. Así, con el primer término se hace referencia a esos casos en los que el estudiante es consciente de que está tomando la información de otra persona y decide no otorgarle los créditos que le corresponden. El plagio inconsciente, por su parte, implica realizar una reproducción de ideas o de palabras que se identifica como una práctica de plagio, pero que se ha producido de forma involuntaria, por desconocimiento o por falta de dominio de los mecanismos de citación (López-Gil y Fernández-López, 2019).

Para entender mejor a qué se hace referencia con el concepto de plagio inconsciente es necesario recuperar el concepto de alfabetización académica. La escritura antes de la universidad consiste, fundamentalmente, en un acto de reproducción de las ideas ajenas. Por el contrario, una vez en la universidad, el alumnado sufre un proceso de alfabetización en el que se enfrenta al reto de dialogar con otros autores y de crear un discurso con una voz propia (Carlino, 2005). Lo que antes se concebía como una realidad única e indiscutible ahora se convierte en una realidad con múltiples miradas. Por tanto, el nivel de intertextualidad requerido es superior al de estadios educativos previos y los estudiantes carecen de las herramientas suficientes para hacer frente a este reto. De hecho, los estudios afirman que es así como lo perciben los propios estudiantes (López-Gil y FernándezLópez, 2019).

Escribir textos en la universidad requiere, entre otras habilidades, ser capaz de localizar la información, acceder a las fuentes y desarrollar 
otra serie de estrategias de documentación (Carlino, 2005). Todas ellas, habilidades que no tienen por qué haber desarrollado en las etapas educativas previas. Así, en ese proceso de alfabetización/apropiación de las reglas de escritura propias de una nueva comunidad discursiva, el estudiante muestra inseguridad y desconocimiento en el uso de las fuentes y en la incorporación de voces ajenas que puede dar lugar a casos de plagio (Ochoa y Cueva, 2014). Por lo tanto, se podrían afirmar que no tienen conciencia de que citar bien no es no tener ideas originales, sino saber utilizar bien las ideas creadas por otras personas (Egaña, 2012). Ruiz (2006) denomina a este fenómeno como dependencia de lo literal. En este sentido, Lillis y Curry (2010) observaron que, en muchos casos, este fenómeno se produciría porque los estudiantes reprimen su voz propia por miedo a que su trabajo sea rechazado.

Como consecuencia, el estudiante al plagiar está haciendo visible una dificultad dentro del proceso de interiorización de la escritura académica o, como se ha mencionado antes, dentro del proceso de alfabetización académica. Dicho de otra manera, los estudiantes universitarios se identificarían como basic writers que no saben aún cómo gestionar su producción escrita (Bizzell, 1986). Se trata de unos escritores que tienen aún retos pendientes asociados al desarrollo de esta habilidad en una nueva comunidad discursiva. Entre ellos se encuentra el aprender a referenciar de manera adecuada las voces que hay tras las ideas que se han planteado. Es precisamente en este contexto en el que nacen los casos de plagio inconsciente.

Frente al estudiantado, además, se encuentra la institución educativa que, en la mayoría de los casos, advierte del peligro del plagio, anuncia las sanciones que puede conllevar su aparición, pero no informa acerca de los mecanismos que tiene el alumnado a su disposición para hacer frente a este problema. Así, entre los desafíos a los que se enfrenta la universidad está el identificar qué prácticas contribuyen al buen flujo y reproducción de la información y formar en ellas a los estudiantes (López-Gil y FernándezLópez, 2019).

El docente, por su parte, tiene un reto doble y es que, además de ser quien va a evaluar los productos producidos por los estudiantes, también tiene entre sus cometidos actuar como facilitador a la hora de introducir al estudiante en la comunidad discursiva. Sin embargo, tienen que ser cuidadosos al ofrecer formas y modelos ya que los previamente mencionados basic writers aprenden a través de la reproducción exacta de los modelos (Bizzell, 1986). Así, la reproducción de las maneras de decir los conceptos, transmitir las ideas, presentar las conclusiones, etc. los haría desempoderarse y, como consecuencia, no desarrollar una voz propia. Por ello, el primero de los pasos que debe dar el docente es cambiar esa idea de 
que los estudiantes plagian porque es fácil hacerlo ya que esa afirmación esconde una falta de comprensión de la complejidad del fenómeno (Sureda, Comas, y Morey, 2009).

En definitiva, el plagio debe entenderse como un constructo social; es decir, como una idea que genera la sociedad. De ahí que el discurso que cada uno tiene sobre el plagio es lo que lo define. Así, tienen lugar tres constructos diferentes: el plagio como cuestión oral, el plagio como elemento regulador y el plagio como aspecto de la escritura académica (Adam, Anderson, \& Spronken-Smith, 2017). Si se entiende el plagio como un aspecto de la escritura académica, sería momento de construir buenas prácticas y de orientar la enseñanza a las necesidades específicas de los estudiantes (Vargas-Franco, 2019). Y es que, tal y como lo perciben los propios estudiantes, las prácticas sancionadoras tienen que ir acompañadas de una aportación didáctica (Ochoa y Cueva, 2014).

\section{MÉTODO}

El trabajo que aquí se presenta es una investigación de tipo cualitativo que está centrada en el análisis de contenido de los productos textuales de carácter académico elaborados por estudiantes de primer curso de grado universitario. A continuación, se presentan las preguntas de investigación que surgen de la revisión teórica, el perfil de los informantes que han participado en el estudio, así como el procedimiento empleado para el análisis de los datos recabados.

\section{Preguntas de investigación}

Como ya se ha adelantado, el objetivo principal de este estudio es, a partir de la premisa de la existencia del binomio plagio inconscienteplagio deliberado, conocer cómo es la naturaleza de los casos de plagio inconsciente que producen los estudiantes una vez están familiarizados con el concepto de plagio. Así, los interrogantes que emergen son los siguientes:

a) ¿Qué naturaleza tienen los casos de plagio inconsciente? ¿Cómo podrían clasificarse?

b) ¿Podría identificarse un patrón de plagio inconsciente entre el alumnado neófito en escritura académica?

c) ¿Qué implicaciones didácticas tendrían la utilización de una taxonomía de plagio inconsciente? 


\section{Perfil de los informantes y contexto de estudio}

En esta investigación ha participado el alumnado de primer curso del Grado de Educación Primaria de una universidad de la Comunidad Autónoma del País Vasco. Un total de 100 estudiantes de dos cursos consecutivos han sido formados a lo largo de 15 semanas en cuestiones asociadas al discurso académico tanto oral como escrito dentro del marco de la asignatura troncal vinculada al trabajo de la competencia comunicativa durante los cursos 2017/2018 y 2018/2019.

Como tarea final de la asignatura han tenido que entregar un texto producido de manera individual. Al final del curso, los estudiantes han participado en un congreso ficticio con una comunicación oral sobre un tema vinculado a su formación pedagógica. Como tarea final, han tenido que escribir un texto de entre 8 y 10 páginas a partir de dicha presentación oral para las actas del congreso (Boillos, 2019). Este documento tendría como base las lecturas sobre diversos temas, concretamente 10 temas diferentes, realizadas a lo largo del curso.

Durante la formación recibida, se dedicaron 7 horas -dos semanas- a la formación específica en cuestiones asociadas al plagio: qué es el plagio, por qué tiene lugar el plagio, el sistema de citación APA, etc. Al finalizar la formación, el alumnado realizó unas tareas de evaluación que permitieron verificar que se habían interiorizado los conocimientos previamente mencionados. Esta formación se impartió íntegramente en lengua castellana y fue en esta lengua en la que se realizaron todas las tareas de la asignatura.

\section{Herramienta de análisis de datos}

El análisis de los textos recabados se ha producido en dos fases. En la primera de ellas, todas las actas han sido filtradas a través del programa Turnitin ${ }^{\circledR}$, una herramienta automática de detección de plagio. El filtro de este software ha dado lugar a unos documentos en formato .pdf en los que aparecía el porcentaje de plagio, así como los fragmentos considerados como tales. En ese documento se indicaba, a su vez, la fuente de la que se habría copiado el texto.

En la segunda de las fases, se ha realizado un análisis de los fragmentos identificados a través de la herramienta para el análisis cualitativo de datos textuales Atlas.ti ${ }^{\circledR}$. Con ella, se han podido establecer categorías de los casos de plagio inconsciente realizados por los estudiantes. Es decir, a partir de los datos recabados, se han ido identificando, de manera inductiva, modalidades de plagio inconsciente que han sido codificadas en tipologías 
y que han sido, posteriormente, agrupadas por categorías. Se considera, por tanto, un estudio diseñado a partir de la metodología Grounded Theory o Teoría Fundamentada (San Martín, 2013) que implica la recolección de datos, su codificación y su posterior reanálisis. Este proceso es el que ha permitido generar una primera aproximación a la taxonomía de casos de plagio inconsciente. Hasta el momento, sí que se han publicado otras taxonomías de plagio (Chowdhury \& Bhattacharyya, 2016), pero estas no contemplan el binomio plagio inconsciente y plagio deliberado.

\section{RESULTADOS Y DISCUSIÓN}

A continuación, se presenta la discusión de los resultados obtenidos en el análisis de datos. Para ello, se retomarán las preguntas de investigación previamente expuestas.

¿Qué naturaleza tienen los casos de plagio inconsciente? ¿Cómo podrían clasificarse?

El análisis pormenorizado de los datos ha permitido constatar que los casos de plagio inconsciente se materializan, fundamentalmente, en dos dimensiones diferentes: a nivel de texto y a nivel del sistema de citación.

A nivel de texto, se encontraría, en primer lugar, la ausencia de reformulación de algunos fragmentos del texto original. Si bien, a priori, podrían parecer casos de plagio deliberado, se han considerado dentro de este grupo cuatro tipos de reproducciones que tienen lugar sistemáticamente en los textos analizados:

- Reproducción de fragmentos breves de texto en los que se expone la metodología o cuestiones de tipo metodológico de una fuente referenciada.

- Reproducción de fragmentos breves en los que se presentan los datos o resultados obtenidos en otras investigaciones.

- Reproducción de objetivos de la misma manera que en la fuente original.

- Reproducción de definiciones de conceptos clave como en la fuente original. 
Parece de interés que ocurran estos fenómenos que vendrían a confirmar la teoría de Egaña (2012) según la cual, los estudiantes no entienden que citar no supone no tener ideas originales, sino saber reproducir y transformar las ideas creadas por otras personas. No sería plagio deliberado porque el resto de la información que acompaña a estos datos sí ha sido reformulada y se ha citado correctamente. Esta casuística también podría vincularse a la dependencia del texto por miedo a tener una voz propia (Lillis \& Curry, 2010).

Otro tipo de fenómeno que ocurre a nivel de texto es el de la modificación de citas marcadas como literales. Se observa el perfil mencionado de los basic writers (Bizzell, 1986) en estos casos en los que los estudiantes citan un fragmento de texto e indican que lo han tomado de manera literal de acuerdo con las normas APA. Sin embargo, ese fragmento que han entrecomillado como literal está modificado, ya sea porque han añadido información, la han modificado o han omitido parte de ella.

En este grupo también se incluirían los ejemplos previamente denominados como patchwriting (McBride, 2012). Esta estrategia consiste en parafrasear parte del texto para que este no sea identificado como fragmento plagiado. Los recursos que emplean los informantes para realizar ese parafraseo son, esencialmente, tres: la adición de información, la modificación y la omisión de parte del texto. Se trataría, por tanto, de los mismos que ya se habían observado en el caso anterior, en la modificación de las citas marcadas como literales. En el caso de la modificación, la estrategia más frecuente es el cambio de un término por un sinónimo y esta se produce indistintamente con verbos, con sustantivos y con adjetivos.

Por último, requieren también atención los casos en los que se reproduce una cita literal de otro texto que se ha leído y se mantiene también la manera en que el autor la introdujo. De ahí que abunden los casos en los que se reconoce como parte del fragmento plagiado el verbum dicenci de una fuente que sí se ha leído. Así, se puede inferir que se ha leído el texto en el que aparece la cita, pero no el texto citado.

Todos estos fenómenos que se producen a nivel de texto presentan, por tanto, distintos grados de manipulación de la fuente original. Así, se podría decir que los casos se encuentran dentro de un continuum de dependencia del texto fuente (Ruiz, 2006). Ello conlleva la identificación de estos fragmentos, por parte de los software, como casos de plagio a pesar de haber indicios de que los autores querían atestiguar la fuente de la que se habían obtenido. 
Por lo que respecta a los casos de plagio inconsciente que se asocian al sistema de citación, se agruparían bajo este epígrafe aquellas reproducciones que afectan a las citas del texto y que han dado lugar a fragmentos identificados como plagio, pero en los que el plagio está provocado por el desconocimiento de la gestión de las fuentes a las que sí se alude. El primero de los tipos es aquel en el que se parafrasean las ideas y, posteriormente, se incluye una misma cita múltiple como en la fuente original. Al igual que ocurría en los casos del verbum dicendi previamente mencionados, esto constata que no se han leído las fuentes originales, sino que se ha accedido a esas ideas de manera indirecta.

Abundan los casos, asimismo, de fragmentos literales que se han citado como no literales y, por tanto, no se han entrecomillado, ni han incluido la página en la que se encuentra dicho fragmento, aunque sí se ha incluido la fuente de la que se ha extraído. En menor medida, también se atestiguan casos en los que se indica el título de la obra, pero no se incluye la cita en el cuerpo del texto tal y como indica la normativa APA.

También se considerarían casos de plagio inconsciente que afectan al sistema de citación aquellas ideas cuya autoría no ha sido debidamente referenciada en el cuerpo del texto por medio de una cita, pero sí aparece la referencia al final. En estos casos, se observa que el estudiante es consciente de que esa idea no era original e indica de dónde la ha obtenido. Sin embargo, no lo hace correctamente en el cuerpo del texto. A pesar de que estos casos no se puedan diagnosticar por medio de un programa de detección de plagio, sí son errores que visibilizan las dificultades en el proceso de alfabetización y que requieren especial atención si se tiene en cuenta que el plagio no es solo una reproducción de palabras, sino también de las ideas (Pecorari, 2008).

Los resultados arriba expuestos llevarían a la creación de una primera tentativa de taxonomía o clasificación que se presenta en la Tabla 1. Serían todas ellas prácticas en las que el plagio no se ha producido de manera deliberada, sino que han venido condicionadas por la falta de dominio del discurso académico; un discurso en el que es necesario reproducir y transformar las ideas de otros autores.

Tabla 1

Taxonomía de casos de plagio inconsciente

P.I. que afecta al texto

Reproducción parcial referenciada
P.I. que afecta al sistema de citación

Multicita 


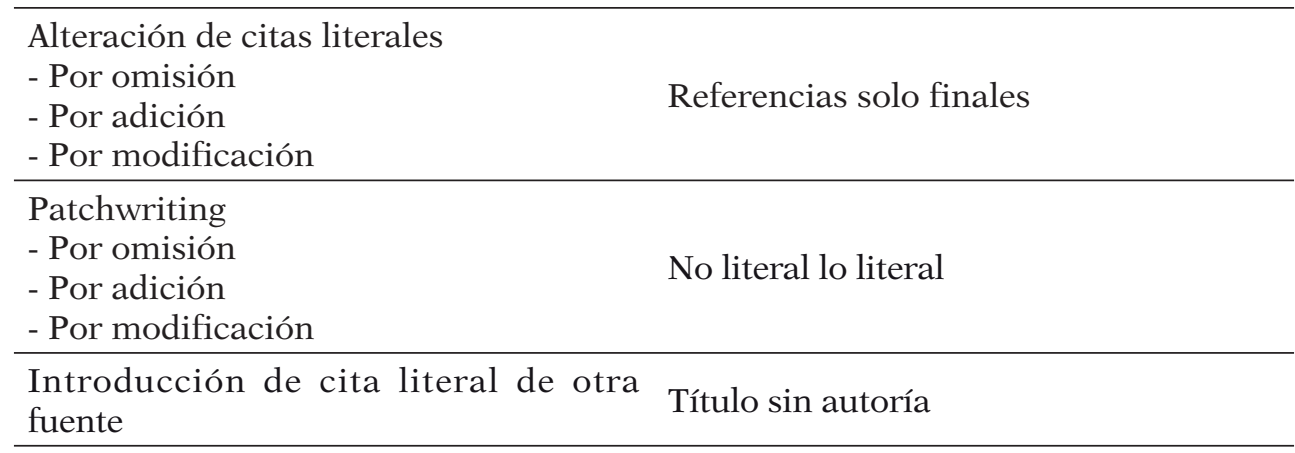

Fuente: elaboración propia

\section{¿Podría identificarse un patrón de plagio inconsciente entre el alumnado neófito en escritura académica?}

Los resultados obtenidos permiten afirmar que, si bien el grado de frecuencia con el que se producen requeriría de un estudio de tipo cuantitativo, sí que se observan tendencias a la reproducción de la misma tipología de errores en la totalidad de los textos que han sido objeto de estudio. La diferencia radica en la frecuencia con la que estos casos se producen. Así, se constatan casos frecuentes de patchwriting o de reproducción de ideas de manera parafraseada sin indicar la autoría. Menos frecuente sería la inclusión del título de la otra sin indicar la autoría.

El dato de enraizamiento de cada uno de los fenómenos según el programa Atlas.ti ® también arroja resultados interesantes en torno a qué tipología de casos de plagio inconsciente tiene mayor protagonismo en los textos. En este sentido, hay una diferencia significativa entre los casos de plagio que afectan al texto y los que afectan al sistema de citación, siendo los primeros los más abundantes. Una vez dentro de este subgrupo, es el patchwriting, como ya se apuntaba, el fenómeno que se da con mayor frecuencia; especialmente, los casos de eliminación parcial de elementos que no impiden que esos fragmentos sean identificados como plagio.

\section{¿Qué implicaciones didácticas tendría la utilización de una taxonomía de plagio inconsciente?}

Una vez identificados los elementos que pueden derivar en plagio involuntario y configurada la clasificación o taxonomía de estos, es momento de reflexionar acerca de cómo tratar estos temas que se puede prever crearán dificultades en los estudiantes. Como se apuntaba, toda actividad sancionadora tiene que ir acompañada de una formación previa 
$\mathrm{y}$, como consecuencia, es necesario generar propuestas que tengan una naturaleza preventiva.

Una de las primeras medidas que debe tomarse es el trabajo de la autoconfianza del estudiante. Si bien la autoexigencia es una herramienta positiva, el estudiante tiene que ser consciente de que está dando pasos en el ese proceso de alfabetización académica en el que está inmerso. Como ya se ha comentado, el estudiante tiene que percibir que tiene algo que aportar a la comunidad científica y que su voz tiene cabida en el discurso que está produciendo (Carlino, 2005).

En este sentido, y de acuerdo con lo planteado por Adam, Anderson, \& Spronken-Smith (2017) el plagio podría considerarse una etapa en el proceso de aprender a escribir en el ámbito académico. En estos textos se ha observado que se dan pasos en el dominio del discurso propio de esta comunidad discursiva cuando hay intentos de referenciar la autoría, cuando se introducen las voces ajenas, etc. Sin embargo, se podría decir que son pasos que atestiguan que los estudiantes se encuentran en un estadio intermedio aún de desarrollo de este nuevo discurso.

Así, una de las primeras herramientas que se pueden emplear para trabajar las dificultades diagnosticadas es el llevar el propio informe obtenido a través de Turnitin ${ }^{\circledR}$-u otra herramienta- al aula, para que los estudiantes comprendan que esas acciones podrían derivar en casos de plagio. Posteriormente, se puede explicar qué acciones realizar en los casos en los que no se ha leído la fuente a la que hace referencia la fuente que sí se han leído. Esto evitaría, entre otros, los casos de multicita o los de cita introducida con el mismo recurso a los que se hacía previamente mención. También sería de interés ofrecer herramientas para el parafraseo que vaya más allá de la mera modificación de palabras por su sinónimo o que supongan la omisión de ciertas palabras del texto original.

También se ha observado que no quedan claros los límites entre lo que es una cita literal y lo que no lo es. Así, es importante explicar que al reproducir una cita literal se debe respetar palabra por palabra lo mencionado por su autor. En este sentido, cabría subrayar que la inclusión de citas literales no quita mérito a su trabajo, sino que demuestran haber sido capaces de hacer un uso estratégico de la información que han recogido en otras fuentes.

No hay que olvidar, tampoco, la necesidad de cambio de los modos en los que se plantean las actividades. En el diseño de las actividades de escritura académica será necesario que los docentes tengan oportunidades para acompañar al estudiante en el proceso de composición. $\mathrm{O}$, como 
apuntaba Bloch (2012), generar actividades en las que los estudiantes pongan en práctica la creación de un discurso académico a través de una voz propia. Estos ejercicios, además de impedir la proliferación del fast food académico al que previamente se hacía mención, van a contribuir a romper la idea preconcebida de que los docentes no se leen los trabajos de los estudiantes (Ochoa y Cueva, 2014). De la misma manera, un análisis de los procesos y no de los productos finales favorecerá el diagnosticar los casos de plagio inconsciente con mayor celeridad.

En definitiva, la escritura debe abordarse como una herramienta para aprender a escribir y no solo desde una perspectiva moral (Adam, Anderson, \& Spronken-Smith, 2017; Childers \& Bruton, 2016). Detrás del plagio existe, por lo general, una falta de aprovechamiento de la dimensión epistémica de la escritura y será labor del docente tomar medidas para poder sacar partido a dicha dimensión. Los docentes no pueden olvidar que escribir es un proceso que posibilita pensar y repensar lo que uno quiere decir, planificar los contenidos, revisarlos, formularlos y reformularlos. La escritura, en tanto comunicación diferida, estimula el análisis crítico sobre el propio saber ya que permite sostener la concentración en ciertas ideas y tiene la potencialidad de ser una forma de estructuración del pensamiento que, al objetivarlo y destinarlo a un otro, lo devuelve modificado (Molina y Carlino, 2013, p.19).

Pero, para que ello tenga lugar, es necesaria la intervención del docente en un rol de guía que ayude a la comprensión de la complejidad del fenómeno al que los estudiantes están expuestos (Sureda, Comas, y Morey, 2009). Sin olvidar que esta tarea no está únicamente en manos de quienes tienen el cometido de formar en escritura académica, sino de todos los docentes en tanto que la alfabetización afecta de manera transversal a todas las asignaturas.

\section{CONCLUSIONES}

En este trabajo se ha llevado a cabo un análisis de las producciones escritas de un grupo de estudiantes universitarios con el fin de identificar qué tipo de prácticas realizan que podrían dar lugar a plagio y cuáles de ellas podrían tipificarse como plagio involuntario. Como resultado del análisis cualitativo de las prácticas recurrentes en todas las producciones, se ha generado una taxonomía que tiene como eje dos tipos de plagio inconsciente: el que afecta al texto y el que atañe al sistema de citación.

Estos fenómenos se han abordado como señales de las dificultades que afrontan los estudiantes a la hora de empezar su proceso de alfabetización 
académica. Se trata de unas dificultades que se manifiestan, en su mayoría, de manera homogénea y que atestiguan un desconocimiento en la gestión de las fuentes directas e indirectas a las que han recurrido para la composición de un nuevo texto. Este hecho es trascendente si se tiene en cuenta que, además de haber recibido formación previa en esta materia, este es precisamente uno de los rasgos que distingue a la escritura académica de las realidades de escritura a las que se ha visto expuesto el alumnado hasta su llegada a la universidad.

De ahí la importancia de llevar a cabo acciones pedagógicas a partir de dicha taxonomía que cumplan dos premisas fundamentales. La primera es que se comprenda que el plagio inconsciente es un indicativo del proceso de adquisición del discurso académico en los estudiantes. La segunda es la necesidad de llevar a cabo buenas prácticas en las que se ponga el foco en los errores que se han presentado en la taxonomía en lugar de intervenir con prácticas sancionadoras que no dan información acerca de dónde está el origen de las dificultades del estudiante. 


\section{REFERENCIAS BIBLIOGRÁFICAS}

Adam, L., Anderson, V., \& SpronkenSmith, R. (2017) 'It's not fair': policy discourses and students' understandings of plagiarism in a New Zealand university. Higher Education, 74(1), 17-32. https://doi.org/10.1007/ s10734-016-0025-9

Amiri, F. \& Razmjoo, S.A. (2016) On Iranian EFL Undergraduate Students' Perceptions of Plagiarism. Journal of Academic Ethics, 14(2), 115-131. https://doi.org/10.1007/s10805-0159245-3

Bizzell, P. (1986). What happens when basic writers come to college? College Composition and Communication, 37(3), 294-301. https://doi. org $/ 10.2307 / 358046$

Bloch, J. (2012). Plagiarism, Intellectual Property and the Teaching of L2 Writing. Multilingual Matters.

Boillos, M.M. (2019). Alfabetización académica temprana a través del aprendizaje basado en proyectos. Lenguaje y textos, (50), 143-154. https:// doi.org/10.4995/lyt.2019.11421

Carlino, P. (2005). Escribir, leer y aprender en la universidad: una introducción a la alfabetización académica. Fondo de Cultura Económica.

Childers, D. \& Bruton, S. (2016). "Should It Be Considered Plagiarism?” Student Perceptions of Complex Citation Issues. Journal of Academic Ethics, 14(1), 1-17. https://doi.org/10.1007/ s10805-015-9250-6

Chowdhury, H.A. \& Bhattacharyya, D.K. (2016). Plagiarism: Taxonomy, Tools and Detection Techniques. 19th National Convention on Knowledge, Library and Information Networking (NACLIN 2016).
Comas, R., Sureda, J., y Oliver, M. (2011). Prácticas de citación y plagio académico en la elaboración textual del alumnado universitario. Education in the Knowledge Society (EKS), 12 (1), 359-385. shorturl.at/wBIR7

Domínguez, D.A., Pérez, M.N., y Quiroz, H.A. (2016). La tecnodependencia, una consecuencia del plagio electrónico en los trabajos académicos: el caso de una universidad privada. Revista Multidisciplinaria de Avances de Investigación, 2(2), 14-29.

Egaña, T. (2012). Uso de bibliografía y plagio académico entre los estudiantes universitarios. Revista de Universidad y Sociedad del Conocimiento (RUSC), 9 (2), 18-30. https://doi.org/10.7238/rusc. v9i2.1209

Hunt, R. (2003). Four reasons to be happy about internet plagiarism. Teaching Perspectives, 5, 1-5.

Ison, D.C. (2015). The influence of the Internet on plagiarism among doctoral dissertations: an empirical study. Journal of Academic Ethics, 13(2), 151166. https://doi.org/10.1007/s10805015-9233-7

Jaramillo, S. y Rincón, N. (2014). Los estudiantes universitarios y la sociedad de la información: una combinación que ha facilitado el plagio académico en las aulas colombianas. Información, Cultura y Sociedad, 30, 127-137.

Lillis, T. \& Curry, M. (2010). Academic Writing in a Global Context: The Politics and Practices of Publishing in English. Routledge.

Linne, J. (2014). "Ahora hago fast food académico". Los estudiantes de la Universidad de Buenos Aires en tiempos de Internet. Palabra Clave, 
17(3), 695-716. https://doi.org/10.5294/ pacla.2014.17.3.7

López-Gil, K.S. y Fernández-López, M.C. (2019). Representaciones sociales de estudiantes universitarios sobre el plagio en la escritura académica. Íkala, Revista de Lenguaje y Cultura, 24 (1), 119-134. https://doi.org/10.17533/ udea.ikala.v24n01a06

McBride, K. (2012). "Pachwriting" is more common than plagiarism, just as dishonest. Poynter. A Global Leader in Journalism.

Molina, M.E. y Carlino, P. (2013). Escribir y argumentar para aprender: las potencialidades epistémicas de las prácticas de argumentación escrita. Texturas, 13, 16-32.

Ochoa, L. y Cueva, A. (2014). El plagio y su relación con los procesos de escritura académica. Forma y Función, 27(2), 95-113. https://doi.org/10.15446/ fyf.v27n2.47667

Pecorari, D. (2008). Academic Writing and Plagiarism: A Linguistic Analysis. Continuum

Real Academia Española (2019). Diccionario de la Real Academia Española.
Ruiz, M. (2006). Dependencia de lo literal y aprendizaje. Textos de didáctica de la lengua y la literatura, 41, 103-114.

San Martín, D. (2014). Teoría fundamentada y Atlas.ti: recursos metodológicos para la investigación educativa. Revista Electrónica de Investigación Educativa, 16(1), 104122.

Sureda, J., Comas, R., y Morey, M. (2009). Las causas del plagio académico entre el alumnado universitario según el profesorado. Revista Iberoamericana de Educación, 50, 197-220.

Sureda, J., Comas, R., \& Urbina, S. (2005). The "copy and paste" generation: plagiarism amongst students, a review of existing literature. International Journal of Learning, 12, 161-168.

Vargas-Franco, A. (2019). Apropiación y plagio académico: un estudio de caso sobre una alumna debutante en la escritura en la educación superior. Íkala, Revista de Lenguaje y Cultura, 24 (1), 155-179. https://doi.org/10.17533/ udea.ikala.v24n01a09

Walker, J. (2010). Measuring plagiarism: Researching what students do, not what they say they do. Studies in Higher Education, 35(1), 41-59. https:// doi.org/10.1080/0307507090291299 


\section{PERFIL ACADÉMICO Y PROFESIONAL DE LA AUTORA} 4724

Mari Mar Boillos Pereira. ORCID: http://orcid.org/0000-0001-5546-

Profesora adjunta. Líneas de investigación: enseñanza de lenguas y formación del profesorado. Email: mariadelmar.boillos@ehu.eus

Fecha Recepción del Artículo: 28. Septiembre. 2019

Fecha Modificación del Artículo: 30. Diciembre. 2019

Fecha Aceptación del Artículo: 11. Enero. 2020

Fecha Revisión para Publicación: 17. Enero.2020 